\title{
Variability of Breast Surface Positioning Using an Active Breathing Coordinator for a Deep Inspiration Breath Hold Technique
}

Kristen McConnell ${ }^{1}$, Neil Kirby ${ }^{2}$, Karl Rasmussen ${ }^{2}$, Alonso N. Gutierrez ${ }^{3}$, Nikos Papanikolaou ${ }^{2}$, Dennis Stanley ${ }^{4}$

1. Department of Radiation Oncology/Medical Physics, University of Alabama at Birmingham, Birmingham, USA 2. Department of Radiation Oncology, University of Texas Health Science Center at San Antonio, San Antonio, USA 3. Department of Radiation Oncology, Miami Cancer Institute, Miami, USA 4. Department of Radiation Oncology, University of Alabama at Birmingham, Birmingham, USA

Corresponding author: Kristen McConnell, kristenmcconnell@uabmc.edu

\section{Abstract}

\section{Purpose}

The Elekta Active Breathing Coordinator ${ }^{\mathrm{TM}}(\mathrm{ABC})$ is used to control breathing and guide deep inspiration breath hold (DIBH). It has been shown to be accurate in lung cancers, but limited analysis has been performed on the spatial accuracy and reproducibility of the breast surface. The use of optical surface-image guidance for patient positioning has grown in popularity and is an alternative solution for breast DIBH. This study aims to evaluate the breast surface variability of an ABC-guided DIBH by using a three-dimensional (3D) surface imaging system to record surface position.

\section{Methods}

Ten participants were placed in the treatment position, and breathing baselines and inhalation volume threshold baselines were monitored and recorded using the $\mathrm{ABC}$. Over 60 minutes, the breathing patterns were recorded by the $\mathrm{ABC}$ and CatalystHD ${ }^{\mathrm{TM}}$ (C-RAD, Uppsala, Sweden). For each breath hold, the valve of the $\mathrm{ABC}$ closed at the baseline inhalation threshold and a 3D surface image was acquired. For each point on the baseline breast surface, a 3D vector was calculated to the subsequent breath hold surface as well as a root mean square (RMS) vector magnitude for the entire surface.

\section{Results}

The average and standard deviation for the RMS difference between the baseline and subsequent evaluated images were $7.12 \pm 2.70 \mathrm{~mm}$.

\section{Conclusion}

Review began 05/28/2021 Review ended 06/02/2021 Published 06/14/2021

\section{() Copyright 2021}

McConnell et al. This is an open access article distributed under the terms of the Creative Commons Attribution License CC-BY 4.0., which permits unrestricted use, distribution, and reproduction in any medium, provided the original author and source are credited.
This study shows that while the ABC-guided inhalation volume is kept constant, a non-negligible variability of the breast surface position exists. Special considerations should be used in clinical situations, where the positioning of the surface is considered more important than inhalation volume.

Categories: Medical Physics, Radiation Oncology

Keywords: dibh, surface imaging, catalysthd, elekta abc, medical physics

\section{Introduction}

When delivering radiotherapy to the left breast, increased cardiac morbidity and mortality are of concern [1]. Deep inspiration breath hold (DIBH) is a technique that has been shown to decrease the mean heart dose while maintaining coverage of the breast [2-8]. In the DIBH technique, a patient performs a breath hold to a specified level during each delivery of radiation. A common choice for controlled DIBH delivery involves using an active breathing coordinator $(\mathrm{ABC})$ system (Elekta, Stockholm, Sweden) to provide information on a patient's predicted location of anatomy. The $\mathrm{ABC}$ utilizes a spirometer-based valve system to control breathing to serve as a guide for DIBH.

The underlying assumption is that a breath hold reaching the same volume indicates consistent internal anatomical positioning. The use of DIBH coupled with the ABC for lung cancer treatment has been shown to reduce critical organ dose [9-11], but there have been studies indicating that lung volume is not a perfect proxy for anatomical position. Plathow et al. showed that lung and chest wall position was dependent on the type of breathing maneuver [12]. The study used dynamic magnetic resonance imaging (MRI) and a fiducial marker to measure the differences in position of the lung and chest wall during three breathing maneuvers: "abdominal breathing", "thoracic breathing”, and "normal breathing”. This could indicate that using the 
Recently, the use of optical surface image guidance has grown in popularity and is an alternative solution for accurate breast DIBH tracking $[13,14]$. With this method, the tracking does not rely on the inspiration volume but rather on the motion of the patient surface. The underlying assumption for this technique, as with all surface imaging systems, is that anatomical location is consistent with the surface location.

Coupling the use of an $\mathrm{ABC}$ to fix the inhalation volume with a surface image of each breath hold has the potential to identify any positioning errors that could result from different breathing maneuvers that manifest during a typical treatment. An abstract published by Flampouri et al. in 2008 used a surface imager coupled with an $\mathrm{ABC}$ to compare and measure inter-breath hold and intra-breath hold [15]. They reported a 2.0-mm surface difference for the inter-breath hold (between two successive breaths) and a 2.5 -mm surface difference for the intra-breath hold (movement within each breath). This study utilized an AlignRT (VisionRT, London, Great Britain) surface imaging system. In our study, 10 participants held their breath to a fixed volume as measured by the $\mathrm{ABC}$. A C-RAD CatalystHD ${ }^{\mathrm{TM}}$ three-dimensional (3D) surface imaging system (C-RAD, Uppsala, Sweden) was used to acquire a surface image during the breath hold, which was subsequently compared to the reference breath hold to measure differences in position.

The abstract of this article was presented at the American Association of Physicists in Medicine (AAPM) Annual Meeting, July 30 to August 3, 2017, Denver, CO.

\section{Materials And Methods}

\section{Elekta Active Breathing Coordinator ${ }^{\mathrm{TM}}$}

The $\mathrm{ABC}$ is a spirometer-based breathing control system consisting of a flexible, disposable mouthpiece connected to a breathing tube, a turbine cartridge to measure volume flow, a balloon valve to control volume flow, a nose clip for limiting air through the nostrils, and a control system [9]. Breathing patterns, both baseline inhalation and breath hold volumes, were established adhering to the recommended standard clinical setup with the $\mathrm{ABC}$ in place.

\section{C-RAD CatalystHD}

The C-RAD CatalystHD is a ceiling-mounted three-camera optical-based imaging system capable of monitoring patient setup and positioning, detecting intra-fraction and inter-fraction motion, and performing respiratory gating. The cameras are mounted at fixed angles so that localization and visualization of the patient are maintained for every gantry location by projecting specific light patterns of a known wavelength (405 nm: near-invisible violet; $528 \mathrm{~nm}$ : green; and $624 \mathrm{~nm}$ : red) onto the patients' skin. The CatalystHD algorithm then matches the light pattern that is projected onto the patients' surface to a reference surface acquired previously (either from the reference CT or from baseline surface scan performed prior to treatment). The CatalystHD algorithm registers the two patterns and provides the patient positioning errors, expressed in six degrees of freedom, required to put the patient into the correct position. Figure $1 a$ shows a representative reference image (green) and Figure $1 b$ shows the real-time patient position (blue) superimposed onto the reference image (green) with calculated shifts for each direction.

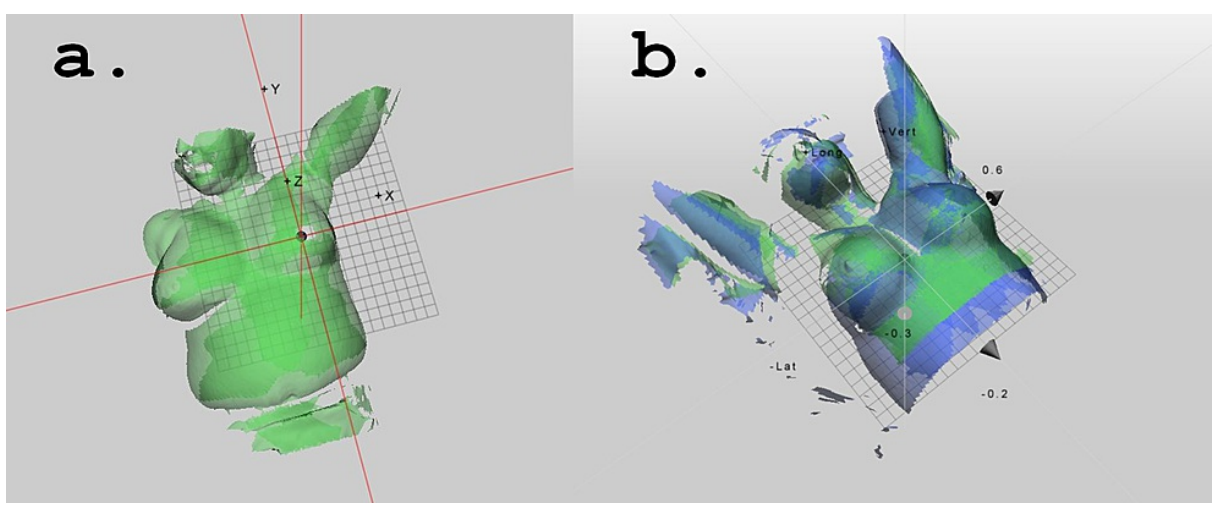

FIGURE 1: (a) Representative reference image (green) and (b) the realtime patient position (blue) superimposed onto the reference image (green) with calculated shifts for each direction.

\section{Study design}

In this study, the $\mathrm{ABC}$ was used to monitor the inspiration volume for 10 female participants (age $=35.1 \pm$ 
12.7 years), while the CatalystHD was used to monitor surface positioning for comparison. To begin, the participants were positioned in a standard clinical left breast DIBH setup using a breast board, set at a standard angle of 10 degrees, with the $\mathrm{ABC}$ system in place. A free-breathing pattern was recorded using the $\mathrm{ABC}$, and then participants were asked to hold their breath to obtain the maximum inspiration volume. Next, a moderate DIBH (mDIBH), set at $80 \%$ of the subject-specific maximum inspiration volume, was established. Figure 2 shows the $\mathrm{mDIBH}$ threshold for a representative patient. This value was used as the trigger for closing the valve of the $\mathrm{ABC}$; once a participant reached that level, the valve would close, signaling the participant to hold their breath.

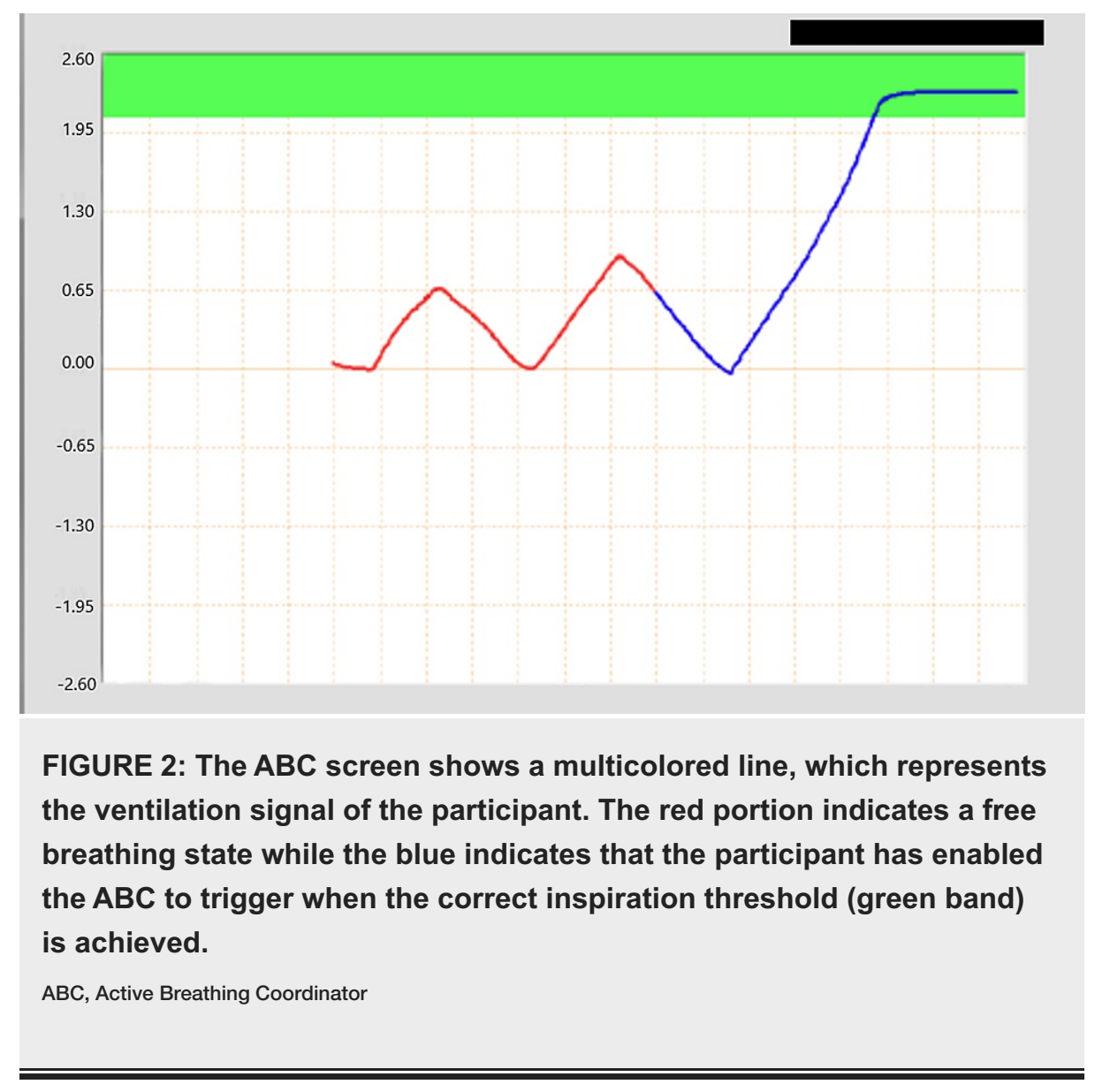

For each participant, during the first breath hold, a reference CatalystHD image was acquired. This reference image served as the baseline with which all subsequent breath hold evaluation images could be compared. Throughout the entirety of the study, a fixed, patient-specific inhalation volume was achieved using the ABC. Simultaneously, the CatalystHD was used to acquire an evaluation 3D surface image. This process was repeated over the course of 60 minutes, providing 30 CatalystHD 3D surface images associated with each subsequent $\mathrm{ABC}$ breath hold. Additionally, as per IRB regulations, the participants of this study were all briefed on the operation of both the $\mathrm{ABC}$ and the CatalystHD and were all familiar with the clinical workflows.

\section{Analysis}

The raw surface images were imported into MATLAB ${ }^{\circledR}$ (MathWorks, Natick, MA), reconstructed as 3D surfaces, and the left breast region of the image was manually contoured on each image and used for analysis. Figure $3 a$ shows the reference breath hold image acquired at the beginning of the study reconstructed as a 3D surface. Figure $3 b$ shows the user-defined breast region of the evaluation image, outlined in red, overlaid on the reference 3D surface image. The selection of this region was made to be representative of the entire clinical breast volume. Figure $3 c$ shows a representative evaluation image with the breast region selected. Figure $3 d$ shows the reference (red) and the evaluation (black) images charted on top of each other. MATLAB was used to calculate the root mean square (RMS) vector error between the reference and evaluation images. For each point on the reference image, a 3D vector was calculated by finding the nearest point on the reference image that matched the closest point on the evaluation breath hold surface. These 3D vectors were used to find an RMS vector error across the entire 3D surface to produce an overall 3D positional error. 


\section{Cureus}

a.

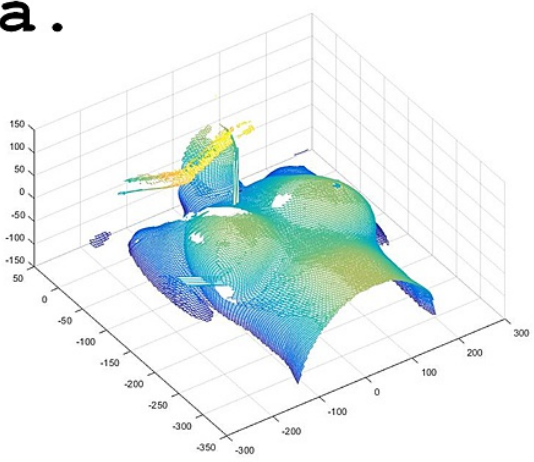

b.

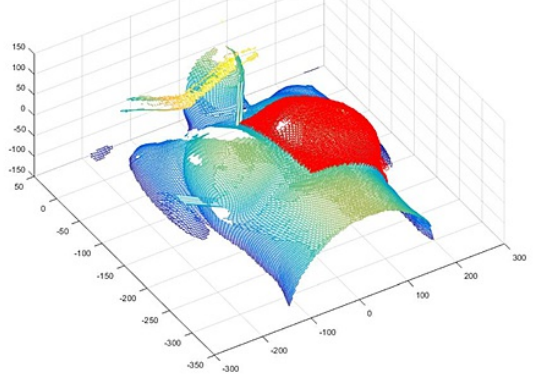

C.

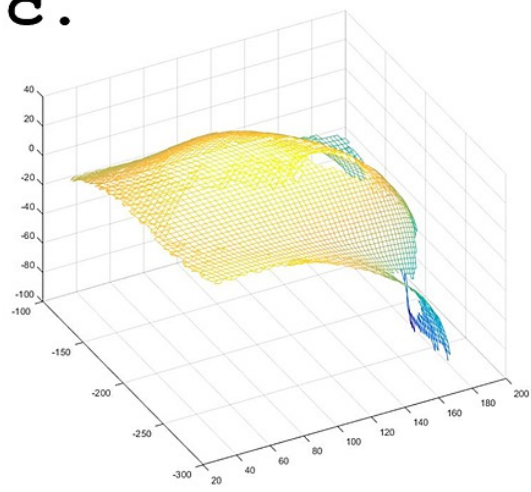

d.

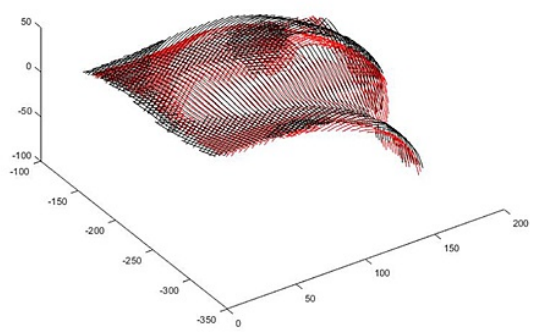

FIGURE 3: (a) Reference breath hold image as a 3D surface, (b) userdefined breast region overlaid on the reference image, (c) representative evaluation image of selected breast region, and (d) reference (red) and evaluation (black) images overlaid.

\section{Results}

Table 1 shows a summary of the average and standard deviation of the 3D RMS difference and normalized inhalation threshold volume. Special care and consideration were taken to ensure that no systematic errors obscured the results. The final 3D error vectors were analyzed to check for any time dependency in an effort to realize any pattern of errors that may arise from patient relaxation during the study. Due to the limited sample size and study regulations designated by the IRB, further investigation of the dependency on breast size is needed.

\section{D RMS Statistical Values}

Average difference (mm)

$7.12 \pm 2.70$

Maximum difference $(\mathrm{mm})$

11.72

Minimum difference $(\mathrm{mm})$

1.02

Median difference $(\mathrm{mm})$

7.67

Normalized inhalation threshold volume (L/L)

$1.0 \pm 0.0$

TABLE 1: Summary of the average and standard deviation of the 3D RMS difference and normalized inhalation threshold volume.

RMS, root mean square

\section{Discussion}

As the technology of radiation therapy and delivery continues to improve, the need for effective and efficient 
motion management has become paramount. Many common treatment sites, such as the breast, lung, and liver, are prone to large target movements and uncertainties as a result of standard respiratory motion. Prior to the availability of motion management techniques, an expansion of the treatment volume was commonly used to account for the uncertainties. As the popularity and availability of motion management systems have increased, management protocols, such as DIBH, have become integral to the reduction of critical organ dose and motion assessment/reduction. The use of the ABC during DIBH techniques has been shown to reduce dose to critical organs [8-11] and is commonly used to control DIBH techniques. Kaza et al. showed that lung volumes controlled with an $\mathrm{ABC}$ were better than when self-controlled during the same session [16]. Consequently, the $\mathrm{ABC}$ can provide reproducible lung volumes, but the underlying assumption for some sites, specifically the breast, is that consistent lung volumes provide consistent positioning. The difference in positioning based on breathing technique suggests that even with a fixed inhalation volume, if a patient changes breathing type, the positioning could change as well.

Alternatively, the use of surface imaging for DIBH has grown recently and provides many advantages including decreased setup time, increased setup accuracy [17], real-time motion management, and no ionizing radiation. Alderliesten et al. showed that using 3D surface imaging for DIBH in patients with leftsided breast cancer was an accurate motion management technique [13]. While surface imaging systems have an inherent deficiency in the ability to visualize internal anatomy, the prevailing benefit is the ability to monitor both the respiratory signal and patient position simultaneously. Using the surface of the patient to monitor, the respiratory signal eliminates the concern that changes in inhalation volume or breathing type could produce a measurable difference in the positioning of the target volume. Additionally, as volumetric modulated arc therapy with a simultaneous integrated boost (VMAT-SIB) for the treatment of breast cancer becomes more prevalent [18-20], the importance of correct positioning of the entire breast surface becomes imperative to accurate dose deposition.

It is important to consider the relevance of motion metrics to the tumor location: volume of air in the lungs for the $\mathrm{ABC}$ or the position of the breast surface in space for the CatalystHD. Our results showed that even though the inspiration volume, as measured by the $\mathrm{ABC}$, was held constant, the images captured by the CatalystHD indicated that, on average, a $7.12 \pm 2.70 \mathrm{~mm}$ difference between the reference and evaluation images existed. Several studies have shown that differences on this magnitude could have potential effects on the toxicity to vital organs at risk, such as the heart and lungs [21-24]. This suggests that the $\mathrm{ABC}$ alone cannot accurately guarantee correct positioning of the breast during DIBH.

\section{Conclusions}

This study showed that even though inhalation volume was kept constant, a non-negligible variability of the breast surface position exists. Special considerations should be used in clinical situations, such as the treatment of breast cancer, where the positioning of the surface is considered more important than inhalation volume.

\section{Additional Information \\ Disclosures}

Human subjects: Consent was obtained or waived by all participants in this study. The University of Texas Health Science Center IRB issued approval HSC20170010H. Animal subjects: All authors have confirmed that this study did not involve animal subjects or tissue. Conflicts of interest: In compliance with the ICMJE uniform disclosure form, all authors declare the following: Payment/services info: The Cancer Prevention and Research Institute of Texas Training Award provided student funding for Kristen A. McConnell (RP140105 and RP 170345). Financial relationships: All authors have declared that they have no financial relationships at present or within the previous three years with any organizations that might have an interest in the submitted work. Other relationships: All authors have declared that there are no other relationships or activities that could appear to have influenced the submitted work.

\section{References}

1. Latty D, Stuart KE, Wang W, Ahern V: Review of deep inspiration breath-hold techniques for the treatment of breast cancer. J Med Radiat Sci. 2015, 62:74-81. 10.1002/jmrs.96

2. Lu HM, Cash E, Chen MH, et al.: Reduction of cardiac volume in left-breast treatment fields by respiratory maneuvers: a CT study. Int J Radiat Oncol Biol Phys. 2000, 47:895-904. 10.1016/s0360-3016(00)00512-5

3. Sixel KE, Aznar MC, Ung YC: Deep inspiration breath hold to reduce irradiated heart volume in breast cancer patients. Int J Radiat Oncol. 2001, 49:199-204. 10.1016/S0360-3016(00)01455-3

4. Remouchamps VM, Vicini FA, Sharpe MB, Kestin LL, Martinez AA, Wong JW: Significant reductions in heart and lung doses using deep inspiration breath hold with active breathing control and intensity-modulated radiation therapy for patients treated with locoregional breast irradiation. Int J Radiat Oncol Biol Phys. 2003, 55:392-406. 10.1016/s0360-3016(02)04143-3

5. Remouchamps VM, Letts N, Vicini FA, et al.: Initial clinical experience with moderate deep-inspiration breath hold using an active breathing control device in the treatment of patients with left-sided breast cancer using external beam radiation therapy. Int J Radiat Oncol Biol Phys. 2003, 56:704-15. 10.1016/s03603016(03)00010-5

6. Pedersen AN, Korreman S, Nyström H, Specht L: Breathing adapted radiotherapy of breast cancer: reduction 
of cardiac and pulmonary doses using voluntary inspiration breath-hold. Radiother Oncol. 2004, 72:53-60. 10.1016/j.radonc.2004.03.012

7. Korreman SS, Pedersen AN, Nøttrup TJ, Specht L, Nyström H: Breathing adapted radiotherapy for breast cancer: comparison of free breathing gating with the breath-hold technique. Radiother Oncol. 2005, 76:3118. 10.1016/j.radonc.2005.07.009

8. Nemoto K, Oguchi M, Nakajima M, Kozuka T, Nose T, Yamashita T: Cardiac-sparing radiotherapy for the left breast cancer with deep breath-holding. Jpn J Radiol. 2009, 27:259-63. 10.1007/s11604-009-0336-1

9. Wong VY, Tung SY, Ng AW, Li FA, Leung JO: Real-time monitoring and control on deep inspiration breathhold for lung cancer radiotherapy--combination of ABC and external marker tracking. Med Phys. 2010, 37:4673-83. 10.1118/1.3476463

10. Sager O, Beyzadeoglu M, Dincoglan F, et al.: Evaluation of active breathing control-moderate deep inspiration breath-hold in definitive non-small cell lung cancer radiotherapy. Neoplasma. 2012, 59:333-40. 10.4149/neo_2012_043

11. Gong G, Wang R, Guo Y, et al.: Reduced lung dose during radiotherapy for thoracic esophageal carcinoma: VMAT combined with active breathing control for moderate DIBH. Radiat Oncol. 2013, 8:291. 10.1186/1748717X-8-291

12. Plathow C, Zimmermann H, Fink C, et al.: Influence of different breathing maneuvers on internal and external organ motion: use of fiducial markers in dynamic MRI. Int J Radiat Oncol Biol Phys. 2005, 62:23845. 10.1016/j.ijrobp.2005.01.042

13. Alderliesten T, Sonke JJ, Betgen A, Honnef J, van Vliet-Vroegindeweij C, Remeijer P: Accuracy evaluation of a 3-dimensional surface imaging system for guidance in deep-inspiration breath-hold radiation therapy. Int J Radiat Oncol Biol Phys. 2013, 85:536-42. 10.1016/j.ijrobp.2012.04.004

14. Cerviño LI, Gupta S, Rose MA, Yashar C, Jiang SB: Using surface imaging and visual coaching to improve the reproducibility and stability of deep-inspiration breath hold for left-breast-cancer radiotherapy. Phys Med Biol. 2009, 54:6853-65. 10.1088/0031-9155/54/22/007

15. Flampouri S, Snead F, Huh S, Symonds-Tayler R, Louis D, Li Z., Palta J: SU-GG-J- 149: position verification of breast treatment with breath-hold technique using 3D-surface and fluoroscopic imaging. Med Physics. 2008, 35:2713-2713. 10.1118/1.2961698

16. Kaza E, Dunlop A, Panek R, et al.: Lung volume reproducibility under ABC control and self-sustained breath-holding. J Appl Clin Med Phys. 2017, 18:154-62. 10.1002/acm2.12034

17. Stanley DN, McConnell KA, Kirby N, Gutiérrez AN, Papanikolaou N, Rasmussen K: Comparison of initial patient setup accuracy between surface imaging and three point localization: a retrospective analysis. J Appl Clin Med Phys. 2017, 18:58-61. 10.1002/acm2.12183

18. Maier J, Knott B, Maerz M, Loeschel R, Koelbl O, Dobler B: Simultaneous integrated boost (SIB) radiation therapy of right sided breast cancer with and without flattening filter - a treatment planning study. Radiat Oncol. 2016, 11:111. 10.1186/s13014-016-0687-6

19. Singla R, King S, Albuquerque K, Creech S, Dogan N: Simultaneous-integrated boost intensity-modulated radiation therapy (SIB-IMRT) in the treatment of early-stage left-sided breast carcinoma. Med Dosim. 2006, 31:190-6. 10.1016/j.meddos.2005.11.001

20. Aly MM, Glatting G, Jahnke L, Wenz F, Abo-Madyan Y: Comparison of breast simultaneous integrated boost (SIB) radiotherapy techniques. Radiat Oncol. 2015, 10:139. 10.1186/s13014-015-0452-2

21. Smyth LM, Knight KA, Aarons YK, Wasiak J: The cardiac dose-sparing benefits of deep inspiration breathhold in left breast irradiation: a systematic review. J Med Radiat Sci. 2015, 62:66-73. 10.1002/jmrs.89

22. Finazzi T, Nguyen VT, Zimmermann F, Papachristofilou A: Impact of patient and treatment characteristics on heart and lung dose in adjuvant radiotherapy for left-sided breast cancer. Radiat Oncol. 2019, 14:153. 10.1186/s13014-019-1364-3

23. Shim JG, Kim JK, Park W, et al.: Dose-volume analysis of lung and heart according to respiration in breast cancer patients treated with breast conserving surgery. J Breast Cancer. 2012, 15:105-10. 10.4048/jbc.2012.15.1.105

24. Pan L, Lei D, Wang W, Luo Y, Wang D: Heart dose linked with cardiac events and overall survival in lung cancer radiotherapy: a meta-analysis. Medicine (Baltimore). 2020, 99:e21964. 10.1097/MD.0000000000021964 